WIENER SLAVISTISCHES JAHRBUCH, Band 52/2006, 213-224

(C) 2007 by Österreichische Akademie der Wissenschaften, Wien

ULRICH THEISSEN

\title{
Die „Passionsblume“ (Passiflora) und ihre Namen in den slawischen Sprachen
}

Kunsthistorisch interessierte Besucher der mittelalterlich-barocken fränkischen Bischofsstadt Bamberg können in der ehemaligen Benediktiner-Abteikirche St. Michael ein einzigartiges Deckenfresko bewundern. Nach einem verheerenden Brand entstand die St. Michaels-Kirche ab 1614 in der heutigen barocken Gestalt, zu der der berühmte Himmelsgarten am gotischen Gewölbe gehört. Die Motive, warum der damalige Abt Johann V. Molitor den Auftrag zur Darstellung von 578 Pflanzen erteilte, sind unbekannt, ebenso ist die Autorschaft unsicher. Möglicherweise waren eigene botanische und pflanzenheilkundliche Interessen der Hintergrund oder die lange gärtnerische Tradition der Stadt Bamberg. Andererseits sind Deckenmalereien mit Pflanzen in mehreren fränkischen und thüringischen Kirchen anzutreffen. Augenscheinlich ist jedenfalls die theologische Symbolik der Malereien im Ganzen und vieler einzelner Pflanzen: Der Garten als Abbild des Paradieses, das Blühen als Sinnbild der Auferstehung, die Anzahl bestimmter Pflanzenteile als Symbol für christliches Gedankengut (Dreifaltigkeit, Pfingsten etc.), die Farbe einzelner Pflanzen. Der Bamberger Apotheker und Naturheilkundler Werner Dressendörfer hat sich durch die botanische Bestimmung und Dokumentation der identifizierbaren Pflanzen, von denen er 100 in einer in der Kirche ausgelegten Planskizze beschrieben hat, unschätzbare Verdienste erworben.

Direkt am Übergang von der Vierung zur Apsis befindet sich in der Mitte die Passionsblume (Passiflora L.), deren Blütenstruktur erstmals von Simone Parlasca (Il Fiore della granadiglia, ovvero della Passione, Bologna 1609) mit den Leiden, im engeren Sinne mit den Marterwerkzeugen Christi in Verbindung gebracht wurde. ${ }^{1}$ Nur fünf Jahre davor hatte Papst Paul V. eine Passiflora incarnata aus Südame-

1 Auffallend ist im Bereich des Vierungsgewölbes eine Häufung von Pflanzen, die einen Bezug zur Passion Christi haben (Dressendörfer 2004: 143). 
rika als Geschenk erhalten (Marzell 1977: 587). ${ }^{2}$ Die mit den in der Folge behandelten serbischen Phytonymen (Simonović 1959: 339) bezeichnete Passiflora caerulea gelangte ebenfalls Anfang des 17. Jahrhunderts aus Peru und Brasilien nach Europa. Ältere Enzyklopädien nennen zumindest für die Passiflora incarnata auch Westindien als Herkunftsland. Meine lexikalischen Beobachtungen lassen mich jedoch fragen, ob die Passionsblume nicht auch für die Flora anderer außereuropäischer Länder typisch war und ist. Im Zusammenhang mit den bulgarischen Phytonymen wurde mir ein persischer Beleg genannt.

Was meinte der Betrachter bzw. was meinten die Betrachter in der Blütenstruktur der Pflanze an biblischem Gedankengut, konkret an Bezügen zur Passion Jesu Christi zu erkennen, die dann zum Benennungsgrund werden konnten? ${ }^{3}$

\begin{tabular}{|l|l|}
\hline \multicolumn{1}{|c|}{ Strukturelement } & \multicolumn{1}{c|}{ Assoziation } \\
\hline Kelch: & \multicolumn{1}{|c|}{} \\
\hline Kronenartiger, weißlich-bläulicher Kelch: & Form: Dornenkrone \\
\hline Blä̈ter: & Farbe: Unschuld \\
\hline Blätter & Hände der Häscher \\
\hline Zehn Blüten- und Kelchblätter (Sepalen) & Zehn treue Apostel (außer Petrus und Judas) \\
\hline Kronblätter (Petalen) & Zerrissene Kleider \\
\hline Dreilappiges Blatt & Lanze \\
\hline Rötlich überlaufene Blätter der Nebenkrone & Vom Blut gerötete Dornenkrone \\
\hline & \\
\hline Blüte: & \\
\hline Oberständiger Fruchtknoten (Ovarium) & In Galle getränkter Schwamm \\
\hline Gestielter Fruchtknoten (Androgynophor) & $\begin{array}{l}\text { Gralskelch, in dem das Blut Christi aufge- } \\
\text { fangen wurde. }\end{array}$ \\
\hline Fünf Staubbeutel (Antheren) & Fünf Wundmale Jesu, Schlagwerkzeuge \\
\hline Drei Blütennarben & $\begin{array}{l}\text { Drei Nägel, mit denen Jesus gekreuzigt } \\
\text { wurde }\end{array}$ \\
\hline & \\
\hline Strahlenkranz (Corona): & Dornenkrone \\
\hline Sprossranken: & Geißelschnüre \\
\hline
\end{tabular}

In der erwähnten Michaelsberger Darstellung der Passionsblume vermischt der Maler phantasievoll die Realität mit diesen Symbolen, wenn er in die Pflanze die geschmiedeten Nägel und die Dornenkrone einfügt (Dressendörfer 1998 und 2004: 143). Die Passiflora war nicht nur ein beliebtes Motiv der christlichen Kunst des Barocks, sondern auch der Romantik, der Fenstermalerei der Neugotik sowie der

${ }^{2}$ Andere Quellen, z. B. Simonović 1959: 339, bezeichnen den Jesuiten J. B. Ferrari († $1653)$ oder in Südamerika wirkende europäische Missionare als Schöpfer dieses Phytonyms und 1633 als Jahr der erstmaligen Erwähnung. Genaust (1996: 462) schreibt den Namen Passiflora jedoch dem spanischen Arzt und Botaniker Nicolás Monardes (14931578) zu. Einem Holzschnitt aus einem Augsburger Einblattdruck von 1609/10 ist zu entnehmen, dass die Pflanze aus Indien stammt (Dressendörfer 2004: 143).

3 Tabelle nach Genaust 1996: 462 und Marzell 1977: 587. 
zeitgenössischen Kunst (Beispiele bei Caesar 1997: 590, hierbei versinnbildlichen Passionsblume und Rose gleichermaßen das Leiden Christi).

Die ikonographische Verbindung der Passiflora mit den Attributen des Martyriums Christi aus der subjektiven Wahrnehmung eines christlich gläubigen Betrachters und Benenners hat nicht nur zum botanischen Namen Passiflora Carl Linnés (1753), auf der Grundlage früherer Bezeichnungen wie Clematis passiflora (bei Abraham Munting), geführt. Dasselbe Benennungsmotiv liegt auch den meisten Bezeichnungen der Passiflora in den europäischen Sprachen zugrunde, die zum Teil erst spät und in Anlehnung an das botanische Taxon entstanden sind.

Auf dieser Grundlage hier eine Auswahl der von Marzell (1977: 587) angeführten Bezeichnungen im deutschen Sprachraum: Jesu Leiden, Leiden Christi, Marter, Passionsblume, Apostelblume, Golgathablume, Herrgottsblume, Kreuzblume, Dornenkraut, Eintagsblume.

Die einzige bei Marzell erfasste Bezeichnung, der ein Benennungsgrund nach objektiven Gegebenheiten der Pflanze zugrunde liegt, ist „Rangapfel“ (offenbar für Passiflora laurifolia oder Passiflora edulis), was durch die Ranken der Gattung motiviert ist. Die folgende Auflistung von Namen für die Passiflora in europäischen Sprachen (außer den hier gesondert untersuchten slawischen) entstammt größtenteils der Suche im Internet und erhebt keinen Anspruch auf Vollständigkeit, da man jede Passiflora-Art eigens nach ihren jeweiligen Namen und deren Synonymen erforschen müsste.

Englisch: passion-flower, passion fruit, maypops, blue crown, granadilla Niederländisch: passiebloem

Französisch: passiflore, fleur de la passion, grenadilles

Italienisch: fiore della passione

Spanisch: $\quad$ pasiflora, pasionaria, flor de pasión

Portugiesisch: maracujá

Rumänisch : pasifloră, floarea-suferínței, ceasornic

Dänisch: passionsblomst

Norwegisch: pasjonsblomst

Schwedisch: passionsblomma, läkepassionblomma

Finnisch: $\quad$ punakas kannatuslill, kärsimyskukka

Ungarisch: $\quad$ golgotavirág (Halász 1970: 1483)

Türkisch: $\quad$ çarkıfelek, firıldakçiçeği (Steuerwald 1972: 169)

Heute ist die Passiflora in ihren unterschiedlichen Arten in erster Linie eine international begehrte und vertriebene Zierpflanze, die inzwischen auch als Zimmerpflanze gut gedeiht. In den Hintergrund tritt dafür ihre Heilwirkung: Für den phar-

\footnotetext{
${ }^{4}$ In älteren Belegen Golgota und auch krisztusvirág (Benkö 1976: 1069).
} 
mazeutischen Gebrauch nennt Berger (1954-1955: 836) 25 Passiflora-Arten, wenn auch die Passiflora incarnata, aber auch ihre sonst eher als Zierpflanze bekannte „blaue Schwester“ Passiflora caerulea, im engeren Sinne als Heilpflanze für folgende Anwendungen gelten:

Die Blätter der Passionsblume (die Wurzel ist giftig) vereinen nach der Klostermedizin die Wirkungen der herzstärkenden Weißdornblätter, der beruhigenden Baldrianwurzel und der krampflösenden Pestwurz in sich. Sie hat sich bei leichten Formen von nervöser Unruhe, Einschlafstörungen, Verspannungen und nervösen Herzbeschwerden bewährt, auch bei Angstzuständen. Ihre wichtigsten Wirkstoffe sind Alkaloide, Flavonoide, Saponine, Cumarinderivate und Maltol (Mayer 2003: 218 sowie http://de.wikipedia.org/wiki/ Passionsblume). Bei Schlafstörungen wird sie in verschiedenen Kombinationen als Tee oder Kapseln verabreicht. Ein Flüssigextrakt (Wasser-Alkohol-Lösung) soll zur Senkung hohen Blutdrucks dienen, in manchen Ländern wird die Passiflora gegen Hyperaktivität und Hysterie bei Kindern eingesetzt. Auch bei Asthma, Depressionen und nervlich bedingten Magen- und Darmbeschwerden soll sie helfen, und das im Gegensatz zur Schulmedizin garantiert ohne Nebenwirkungen. Seltenere Anwendung erfährt die Passiflora bei Augenentzündungen und Sehstörungen. Gefährlich kann sie allein in überhöhter Dosis als gerauchte Droge werden, wobei ihre Wirkung der des Marihuana ähnlich sein soll. ${ }^{6}$ Schon die Indianer, die die Passiflora zuerst anbauten, wussten ihre Wirkung zu schätzen, wobei sie allerdings die Wurzel verarbeiteten (Caesar 1997: 588).

Ihre heutige Popularität ${ }^{7}$ verdankt die Passionsblume weniger den Heilwirkungen der Kloster- und der homöopathischen Medizin als der ,exotischen“ und in vitaminreichen Fruchtdrinks sowie in Eis enthaltenen Frucht der Pflanze, die ursprünglich reine Obstpflanze war und schon vor Ankunft der Europäer von den Indianern angebaut wurde. Auf dem amerikanischen Kontinent wachsen etwa 400 bis 600 Passiflora-Arten, von denen etwa 50 bis 60 essbare Früchte tragen (Caesar 1997: 587). Bei der Passiflora murucuja und der Passiflora edulis handelt es sich um die Maracuja oder Grenadille (granadilla ${ }^{8}$ ), eben auch als Passionsfrucht bekannt.

Die meisten europäischen Sprachen haben, wie oben gezeigt, ihren Namen für die Passiflora aus der Botanik entlehnt. Interessanter und vielfältiger wird es, wenn wir das deutsche Sprachgebiet in Richtung Osten und Südosten verlassen, um zu sehen, wie diese Pflanze in slawischen Sprachen benannt wird und welche Gründe zu dieser Benennung geführt haben könnten:

\section{$\mathrm{B} \mathrm{u} l \mathrm{~g}$ a ri s c h}

In dem sechssprachigen Heilpflanzenwörterbuch von Liljana Christova (1987) ist die Passionsblume trotz ihrer Bedeutung für die Volksmedizin nicht vertreten.

5 Das Artepithethon incarnata ,die Fleisch Gewordene“ nimmt ebenfalls Bezug auf den menschgewordenen Christus.

6 http://www.health-marketplace.com/Passion-Flower.htm - Schunk 1996: 52.

7 Die internationale „Passiflora-Lobby“ ist auch medial präsent: http://www.passiflora.org im Hinblick auf die Vielfalt der Passiflora-Arten lohnt sich ein Blick auf http:// www.passiebloem.nl

${ }^{8}$ Hier handelt es sich um die Deminutivform von granada 'Granatapfel'. 
Shekow (2003: 88) erwähnt für die Passiflora incarnata die Entlehnung des Gattungsnamens пасифлора. Dieser bezeichnet bei Kitanov (1994: 52) die Passiflora quadrangularis, die bei ihm deutsch als „Melonengranadille“ und „Königsgranadille" wiedergegeben ist. Interessanteren Aufschluss bietet ein Blick in ältere Quellen der bulgarischen Phytolinguistik, deren Anfänge von Botanikern, nicht von Philologen, gelegt wurden. ${ }^{9}$

1. Звезда, офицерска (MBBR 1939: 229)

Mit dem Element „Stern“ wurden und werden im Bulgarischen zahlreiche Pflanzennamen gebildet (nach BER I: 621):

\begin{tabular}{|c|c|c|}
\hline алпийска звезда & Gnaphalium & ,,Ruhrkraut ${ }^{“ 6}$ \\
\hline балканска звезда & Leontipodium alpinum & „Edelweiß“ \\
\hline водна звезда & Callitriche vernalis & „Sumpfwasserstern“ \\
\hline жълта звезда & Gagea lutea & „Waldgoldstern“ \\
\hline горска звездица & Stellaria holostea & „Sternmiere“ \\
\hline бяло звездиче & Dorycnium herbaceum & „Krautiger Backenklee“ \\
\hline звездан & Lotus corniculatus & „Gemeiner Hornklee“10 \\
\hline \multirow[t]{4}{*}{ звездел } & Lotus corniculatus & „Gemeiner Hornklee“" \\
\hline & Trifolium & ,Klee ${ }^{612}$ \\
\hline & Meliotus officinalis & „Echter Honigklee“ \\
\hline & Medicago lupulina & „Hopfenklee“ \\
\hline
\end{tabular}

Ein Blick auf die Blüte der Passionsblume ermöglicht neben allen christlichen Assoziationen auch jene mit einem Stern.

\section{2. Марта (MBBR 1939: 229)}

Das Phytonym марта ist von март („März“) abgeleitet. Bei Gerov (III: 51) ist die feminine Form noch der Standard für den Namen des Monats, der im Zusammenhang mit dem Feiertag des Frühjahrsanfangs als alte Frau personalisiert wurde (Честита Баба Марта!). Der BER (III: 672-673) verzeichnet für марта allerdings nicht „Passionsblume“ als Bedeutung, sondern Bellis perennis „Gänseblümchen“ und Tussilago farfara „Huflattich“ (bei Gerov жълта марта. Бяла марта bezeichnet die Margerite Chrysanthemum leucanthemum). Weitere Pflanzenbezeichnungen, zu

\footnotetext{
9 Die Schreibung wurde der heutigen bulgarischen Orthographie angeglichen.

10 Gerov II: 146, Shekow 2003: 77. Der ,gemeine Hornklee“ wirkt als Heilpflanze ähnlich wie die Passionsblume (Bremness 1994: 184) - das hat allerdings nichts mit dem in den beiden Phytonymen gemeinsamen Element „Stern“ zu tun.

11 Shekow 2003: 77.

12 Gerov II: 146.
} 
denen der BER nur begrenzt botanische Termini anführt, sind мартинче (бяло копито) und диво мартинче (пачи крак Gagea „Gelbstern“).

Für das Makedonische verzeichnet Koneski (1986: 402) zwar das Phytonym бела марта, allerdings mit der Bedeutung Tussilago farfara „Huflattich“.

\section{3. Мушник (MBBR 1939: 229)}

Der BER (IV: 367) sieht über die gemeinsame Pluralform мушници einen $\mathrm{Zu-}$ sammenhang zwischen мушник und мушница, was „für den Winter getrocknete grüne Bohne" bedeutet (bei Gerov III: 94 ist als Zweitbedeutung чушка angegeben). Die Hauptbedeutung sei aber eine „geflochtene Geldtasche für Frauen“. Мушница, eine Augmentativbildung mit dem Suffix -ица, ist in seiner Grundform schon im Altbulgarischen belegt (ношьна „Tasche, Beutel“) und in weiteren heutigen Slawinen verbreitet (slowenisch môšnjica, russisch мошница und tschechisch mošnička). Der Pflanzenname мушник könnte jedoch auch eine Ableitung vom Verb муша bzw. мушвам/мушна „stechen (mit dem Messer), stoßen (mit den Hörnern)“ sein, was im Zusammenhang mit der Corona der Blüte ein durchaus plausibler Benennungsgrund wäre.

\section{4. Сахат-чиче, сахатче, синьо сахатче}

Die Herausgeber der MBBR (1939: 229-230) identifizieren сахат-чиче ${ }^{13}$ und чаргифилекъ als eindeutig türkischen Ursprungs. Mladenov (1941: 570) weist auf das Arabische als Ursprungssprache hin. Diese Angaben werden durch den BER (VI: 516) bestätigt: сахат bedeutet im Arabischen ( $\left.s \bar{a}^{\prime} a t\right)$ eine „kurze Zeitspanne, einen Moment“, aber auch „eine Stunde“ und gar „die Uhr“, in der Vermittlersprache Türkisch (saat, ältere Form sahat) „Stunde; Uhr“. Dieses Wort wurde auch ins Serbische, Kroatische, Albanische und Aromunische entlehnt. Das Rumänische bietet eine Übersetzung dieses Namens als phonetisch adaptierte Entlehnung aus dem Bulgarischen: ceasornic < часовник, s. u. Unter den zahlreichen deminutiven Ableitungen im Bulgarischen findet sich auch das Phytonym сахатче in mehreren morphologischen Varianten, die den Schierlings-Reiherschnabel Erodium cicutarium, die Hauhechel Ononis spinosa, und zusammen mit dem Adjektivattribut синьо auch die Passiflora caerulea bezeichnen.

Unter dem Lemma сахатчя nennt Gerov (V: 121) noch die wenig poetische und zweifellos durch die osmanische Herrschaft motivierte Bezeichnung вий-вийкараджа (,heule-heule-Karadža“). Derartige imperativisch gebildete Pflanzennamen sind im Bulgarischen sonst eher im Zusammenhang mit Tieren anzutreffen (etwa мяу-коте für den Gartensalbei Salvia officinalis oder лай-лай-кучка für die Kamille Matricaria chamomilla).

${ }^{13}$ In dieser Schreibung auch bei Grannes 2002: 227. 
Zur Motivation des Namens сахатче und seiner Varianten verweise ich auf die Ausführungen zu seiner bulgarischen Entsprechung часовник.

5. Срастниче, срастоцвет [Druckfehler statt страстоцвет?] (MBBR 1939: 229230)

Der Band VII des BER ist noch nicht erschienen, so dass wir keine etymologische Quelle zur Bedeutung dieser Namen konsultieren könnten. Auch Mladenov führt sie nicht an. Geht man davon aus, dass die Schreibung korrekt ist, ließe sich rein etymologisch eine Ableitung von der Wurzel *rast „,wachsen“ bzw. vom Verb сраствам/срасна „zusammenwachsen“ konstruieren.

Die bei Gerov (V: 535) angeführte Bezeichnung страстоцвет (beim Lemma чаргифилек) bietet einen Hinweis darauf, dass es sich bei den vom MBBR angegebenen Phytonymen um fehlerhafte Schreibungen (Auslassung des T) handelt. Wir hätten es bei с(т)растниче und с(т)растоцвет demnach mit einer Lehnübersetzung von Passi-Flora als einer „Leidensblume“ zu tun.

6. Чаргифилек (MBBR 1939: 230), in anderer Schreibung чарк-фелек (Grannes 2002: 280).

Dieser Name steht völlig isoliert da und hat allenfalls noch Bedeutung für die historische Dialektologie des Bulgarischen. War der bulgarische Wortschatz zu Zeiten Najden Gerovs und der vorbereitenden Forschungen zu den MBBR noch stark vom Osman-Türkischen geprägt, so sagt heute чаргифилек nicht einmal mehr botanisch gebildeten Bulgaren etwas. Es handelt sich um ein Lehnwort aus dem Persischen (çarx-i falak), das über das Türkische (çarklfelek) ins Bulgarische gelangt ist. Als Bedeutungen gibt Steuerwald (1972: 169) an: 1. Himmelsgewölbe, 2. wechselndes Schicksal, Rad des Schicksals, 3. Feuerrad, Feuersonne, 4. blaue Passionsblume Passiflora caerulea. Die Motivation des Pflanzennamens ist aus der Kenntnis der Blütenstruktur und den Bedeutungen 1-3 gut nachvollziehbar: Benennungsgrund waren die leuchtenden Farben und die runde Form des Strahlenkranzes. ${ }^{14}$ Ein Lehnwort aus dem Persischen? Sollte Südamerika doch nicht das alleinige Herkunftsland der Passiflora sein?

Gerov (V: 535) bietet unter чаргифилек für die Passiflora caerulea noch die Synonyme кавалерник und страстоцвет. Zum möglichen Benennungsmotiv des Namens кавалерник siehe oben unter офицерска звезда.

\footnotetext{
${ }^{14}$ Für wertvolle Hilfe aus turkologischer Perspektive danke ich herzlichst Herrn Prof. Semih Tezcan, Universität Bamberg.
} 
7. Часовник (MBBR 1939: 230).

Ilčev (о. J.: 180) erwähnt zudem das Deminutivum часовниче, das in anderen Quellen (Shekow 2003: 56) den Schierlings-Reiherschnabel Erodium cicutarium bezeichnet.

Часовник 'Uhr' ist die bulgarische Entsprechung zum arabisch-türkischen Wort сахат (s. o.), das auch dem serbisch-kroatisch-bosnischen sât zugrunde liegt (Škaljić 1989: 542). Um den Zusammenhang mit einer Uhr als Benennungsmotiv des Betrachters nachvollziehen zu können, gilt es, einen genaueren Blick von oben auf die Blüte der Passionsblume zu werfen, wobei die gegeneinander versetzten drei Griffel über den fünf Staubbeuteln durchaus als Zeiger einer Uhr gesehen werden könnten.

Es ist zu erwähnen, dass der Turzismus сахат $>$ часовник als ceasornic in der Bedeutung Passiflora ins Rumänische entlehnt wurde (Seche 1982: 121, Tiktin 1986: 492).

\section{$\mathrm{Kr}$ o a t i s c h}

Mukokaz, kristovo cvijeće (Poljanec 1973: 1135). Erstaunlicherweise sucht man mukokaz in dem aktuellen ausführlichen Wörterbüchern des Kroatischen (Anić und RHJ) vergeblich. Das Fremdwörterbuch von Anić - Goldstein (1999: 524) nennt die Phytonyme kristov cvijet und gospodinova kruna.

$\mathrm{O}$ b e r s o r b i s c h

Ćerpjeńka, ćerpjenna kwětka, matrownica (Rězak 1920: 727). Auch hier steht der Pflanzenname semantisch mit dem Dulden und Leiden, mit der Passion Christi in Verbindung. Bei matrownica handelt es sich um eine Variante des Namens martrownica und damit teilweise um eine Entlehnung des deutschen Wortes Marter. Im obersorbischen Wörterbuch von Pfuhl (1954: 354) findet sich das Phytonym boža martra, also 'Gottesmarter' für das 'Mutterkraut' Chrysanthemum parthenium - so jedenfalls laut Pfuhl.

$\mathrm{P}$ o l n i s c h

Męczennica (krwista), kwiat męki Pańskiej (SJP III: 484) ${ }^{15}$. Męczennica bedeutet eigentlich 'Märtyrerin'. Die blaue Passionsblume Passiflora caerulea heißt męczennica btękitna (Zimny 1994: 825-826).

Rus s is ch

Das in den meisten Wörterbüchern und in botanischer Spezialliteratur am häufigsten auftretende Phytonym für die Passiflora ist die Lehnübersetzung страстоцвет. Eine „ausführlichere“ Form ist цветок страстей господних. Die MBBR (1939: 230) erwähnen für das Russische auch die Bezeichnung кавалерская звезда, die ich

${ }^{15}$ vgl. http://www.flora.moo.boo.pl 
jedoch in den gebräuchlichen Wörterbüchern nur bei Vladimir Dal' (1955: 673) finden konnte. Hingegen scheint dieser ,,säkularisierte“ Name inzwischen ein gängiges Synonym zu sein, geht man von seiner Frequenz im Internet aus. Jedenfalls lässt der Blick auf die sternförmige Blütenstruktur auch diese Benennungsmotivation schlüssig erscheinen. Annenkov nennt in seinem Wörterbuch (1878: 243) noch die Varianten страстоцветка, кавалерник und лианы. Der Name венец Христов ist aus einer älteren Quelle übernommen (Kondratovič 1780). ${ }^{16}$

$\mathrm{S}$ e r b i s c h

Simonović (1959: 339) und Ristić - Kangrga (1963: 172) führen für das Serbische (bei letzteren für das Serbokroatische) folgende Entsprechungen von Passiflora coerulea an, die ohne Ausnahme eng im Zusammenhang mit der Leidensgeschichte Christi stehen bzw. mögliche Lehnübersetzungen aus anderen Sprachen sind:

$\begin{array}{ll}\begin{array}{l}\text { господинова круница } \\ \text { пасионски цвет }\end{array} & \text { 'Herrenkrone' } \\ \text { пасифлора } & \text { 'Passionsblume' } \\ \text { христов венац } & \text { 'Christi Kranz', }\end{array}$

Auch der Name богородичин крст 'Muttergotteskreuz' steht in engem Zusammenhang mit der Leidensgeschichte Jesu. Er erinnert an die in der biblischen Überlieferung nicht thematisierten angesprochenen Leiden der Gottesmutter unter dem Kreuz ihres Sohnes, die erst in der Sequenz „Stabat mater dolorosa“ des Franziskaners Jacopone da Todi (1230-1306) eine literarische Bedeutung bekommen sollten.

S 1 o w a k i s h

Mučenka (Čierna 1986: 524).

S low e n i s c h

Pasijonka, trpljenka (Debenjak 1993: 823). Im slowenisch-deutschen Teil ihres lexikographischen Chef-d'oeuvre führen die Geschwister Debenjak neben den Arten trpljenka modra und trpljenka užitna auch das Phytonym barbadinka für die „Riesengranadilla“ (?) an, dessen genaue etymologische Deutung mir derzeit nicht möglich ist. Man findet es nicht einmal in den slowenischen Suchmaschinen des Internet.

$\mathrm{T} \mathrm{s} \mathrm{c} \mathrm{h} \mathrm{e} \mathrm{c} \mathrm{h} \mathrm{i} \mathrm{s} \mathrm{c} \mathrm{h}$

Mučenka, pasiflora. Haller (ČSVS I: 170) führt noch die volkstümlichen Formen boži muka und boži umučení an.

${ }^{16}$ Annenkov nennt auch mehrere georgische Bezeichnungen für die Passionsblume, die hier linguistisch weniger von Belang, aber im Hinblick auf Herkunft und Verbreitung dieser Pflanze durchaus von Interesse sind. 
Der Pflanzenname mučenka ist vom Verb mučit 'quälen, foltern' abgeleitet. Weder dieses ebenfalls an die Leiden Christi erinnernde Phytonym noch der botanische Gattungsname Passiflora sind im führenden Werk Václav Macheks über die tschechischen und slowakischen Pflanzennamen vertreten. Es ist denkbar, dass die Passionsblume, die heute auch in Tschechien beliebte Zier-, Genuss- und Heilpflanze ist, in der Flora der damaligen Tschechoslowakei einfach aus klimatischen Gründen nicht vertreten war oder von Machek übersehen wurde. Gebauer erwähnt sie für das Alttschechische jedenfalls nicht. Heute lässt sich selbst bei einer Online-Suche nur der Gattungsname mučenka mit zahlreichen Artepitheta ausmachen, bei denen es sich ebenfalls um Lehnübersetzungen des Artennamens der botanischen Bezeichnung handelt (modrá $=$ caerulea, pletni $=$ incarnata, žlutá $=$ lutea $)$.

\section{$\mathrm{Uk} \mathrm{ra} \mathrm{in} \mathrm{i} \mathrm{s} \mathrm{c} \mathrm{h}$}

Пасифлора, мучениця (RUS 1984: 459). Die ukrainische Bezeichnung мучениця, ebenfalls eine Teilentlehnung aus Passiflora, sucht man im I. Band des großen Slovnik ukrains'koj movi vergeblich.

W e i $\beta$ r u s s i s c h

Пасифлора, страстоцвет (TSBM 1980: 459). Ob das Weißrussische und das Ukrainische wohl je eigene Namen für die Passionsblume hatten?

Es wird klar, dass es sich bei den slawischsprachigen Bezeichnungen für die Passiflora zum größeren Teil um Übersetzungen dieses in Südeuropa (Italien oder Spanien) geprägten botanischen Namens handelt bzw. um Namen, die den Inhalt dieses Begriffes, also den Märtyrertod Christi, bis hin zur Trauer seiner Mutter (богородичин крст im Serbischen) umschreiben. Das Benennungsmotiv, die persönlichen Assoziationen eines gläubigen und theologisch geschulten Betrachters und Benenners, ist also gleichsam eine europäische Universalie. Bei den anderen Phytonymen, wie sie vor allem im Bulgarischen (unter Einfluss der osmanischem Kultur) und im Russischen zu finden sind, waren die Blütengestalt der Passionsblume und ihr subjektiv hergestellter Bezug zum Firmament oder zu Alltagsgegenständen (Uhr) und militärischen Symbolen (Sterne) ausschlaggebend. Wolfgang Caesar (1997: 587) behauptet, es gelte bei allen Völkern gleichsam eine „Faustregel“, dass die Pflanzen primär nach ihrem Nutzen und erst sekundär nach ästhetischen und symbolischen Gesichtspunkten benannt werden. Dem ist nicht nur im Fall der Passiflora entschieden zu widersprechen. Ich konnte bei meiner Untersuchung jedenfalls keinen ,nutzenorientierten“ Pflanzennamen ausmachen, der nur irgendwie mit den oben erwähnten nachgewiesenen Heilwirkungen der Passiflora zu tun hätte. 
BER I, III, IV, V:

ČSVS I:

MBBR 1939:

NRBS 1971:

OSN 1902:

RHJ 2003:

RUS II:

SJP III:

TSBM 1980:

Anić 1998:

Anić - Goldstein

Annenkov 1878

Benkö 1967:

Berger 1954-1955:

Bremness 1994:

Caesar 1997:

Čierna 1986:

Dal' I:

Debenjak 1993:

Debenjak 1995:

Dressendörfer 1998:

Dressendörfer 2004:

Gebauer 1916:

Genaust 1996:

Gerov I, III, V:

Grannes 2002:

Halász 1970:

Ilčev o. J.:

Kitanov 1994:

Koneski 1986:

Kozarov 1925:
Български етимологичен речник. I. A-3 (1971), III. крес - минго̀ (1986), IV. минго̀ - па̀дам (1995), V. пускам-словар². VI (2002), София

Český slovník věcný a synonymický I, Praha 1969

Б. Давидов, А. Явашев, Б. Ахтаров (Hrsg.), Материали за български ботаничен речник, София

В. И. И. Синягин, О. И. Чибисова (Hrsg.), Немецко-русский биологический словарь, Москва

Ottův slovník naučný 19: P-Pohoř, Praha

Rječnik hrvatskoga jezika, Zagreb

Русско-украинский словарь II: н-приять (1979), Киев

Słownik języka polskiego. Tom III (1981), Warszawa

Тлумачальны слоўник беларускай мови. Том 4: П-Р, Минск

V. Anić, Rječnik hrvatskoga jezika, Zagreb

V. Anić, I. Goldstein, Rječnik stranih riječi, Zagreb

Н. Анненков, Ботанический словарь, Санктпетербург

L. Benkö, A magyar nyelv tortenéti-etimológiaí szótára I: A-Gy, Budapest

F. Berger, Synonyma-Lexikon der Heil- und Nutzpflanzen, Wien

L. Bremness, Kräuter, Gewürze und Heilpflanzen, Ravensburg

W. Caesar, R. Spohn, Passionsblume. Kulturhistorische Aspekte einer Heilpflanze, Deutsche Apotheker Zeitung, 137. Jahrgang, Nr. 8, 587593

M. Čierna, E. Geze, M. Juríková, E. Menke, Nemecko-slovenský slovník. Deutsch-slowakisches Wörterbuch, Bratislava

В. Даль, Толковый словарь живого великорусского языка. Том I: А-3, Москва

D. Debenjak, Veliki nemško-slovenski slovar. Großes deutsch-slowenisches Wörterbuch, Ljubljana

D. Debenjak, Veliki slovensko-nemški slovar. Großes slowenischdeutsches Wörterbuch, Ljubljana

W. Dressendörfer, Der Himmelsgarten an der Decke von St. Michael [Bamberg]. Mit einem Lageplan für 100 Pflanzen, 3. Auflage

W. Dressendörfer, Die Pflanzenbilder im „Himmelsgarten“ von St. Michael zu Bamberg - eine Annäherung, in: W. Taegert (Hrsg.), Hortulus Floridus Bambergensis. Studien zur fränkischen Kunst- und Kulturgeschichte. Renate Baumgärtel-Fleischmann zum 4. Mai 2002, Petersberg

J. Gebauer, Slovník staročeský, Praha

H. Genaust, Etymologisches Wörterbuch der botanischen Pflanzennamen, Basel - Boston - Berlin

Н. Геров, Речник на българския език. Фототипно издание. I-VI (1975-1978), София

A. Grannes, K. Rå Hauge, H. Süleymanoğlu, A dictionary of Turkisms in Bulgarian, Oslo

E. Halász, Német-magyar szótár II, Budapest

Ст. Илчев, Ботаничен речник, София

Б. Китанов, Ботанически речник. Български - латински - руски английски - френски - немски, София

Б. Конески, Речник на македонскиот јазик со српскохрватски толкувања, Скопје

П. Козаров, Български народни названия на растенията, София 
Kuechen 1989: $\quad$ U.-B. Kuechen, Das späte Eindringen der Passionsblume in den ,,mundus symbolicus“, in: Klaus Matzel (Hrsg.), Festschrift für Herbert Kolb zu seinem 65. Geburtstag, Bern, 361-388

Machek 1954:

Marzell 1977:

V. Machek, Česká a slovenská iména rostlin, Praha

H. Marzell, Wörterbuch der deutschen Pflanzennamen. Dritter Band: Macleya-Ruta, Stuttgart - Wiesbaden

Mayer 2003:

J. G. Mayer, B. Uehleke, P. K. Saum, Handbuch der Klosterheilkunde. Neues Wissen über die Wirkung der Heilpflanzen. Vorbeugen, behandeln und heilen, München

Mladenov 1941: Ст. Младенов, Етимологически и правописен речник на българския книжовен език, София

Pfuhl 1954:

C. T. Pfuhl, Obersorbisches Wörterbuch. Fotomechanischer Neudruck, Bautzen

Poljanec 1973: $\quad$ R. F. Poljanec, S. M. Machatova-Poljanec, Rusko-hrvatski rječnik, Zagreb

Rězak 1920: $\quad$ P. Rězak, Němsko-serbski wšowědny słownik hornjołužiskeje rěče. Deutsch-wendisches enzyklopädisches Wörterbuch der oberlausitzer Sprache, Bautzen

Ristić 1963:

S. Ristić, J. Kangrga, Enciklopedijski nemačko-srpskohrvatski rečnik. Enzyklopädisches deutsch-serbokroatisches Wörterbuch, München Beograd

Schunk 1996:

R. Schunk, Heilkraft aus Heilpflanzen, Abtswind

Seche 1982:

L. Seche, M. Seche, Dictionarul de sinonime al limbi române, Bucureşti

Simonović 1959:

Snoj 1997:

Steuerwald 1972:

Д. Симоновић, Ботанички речник, Београд

Škaljić 1989:

Šulek 1879:

Tiktin 1986:

Zimny 1994:

M. Snoj, Etimološki slovar slovenskega jezika, Ljubljana

K. Steuerwald, Türkisch-deutsches Wörterbuch, Wiesbaden

A. Škaljić, Turcizmi u srpskohrvatskom jeziku, Sarajevo

B. Šlek, Jugoslovenski imenik bilja, Zagreb

H. Tiktin, P. Miron, Rumänisch-deutsches Wörterbuch, Wiesbaden

L. Zimny, Deutsch-polnisches Landwirtschafts-Wörterbuch. Wielki słownik rolniczy niemiecko-polski, Wrocław

Ulrich Theißen

Fachbereich Slawistik der Universität Salzburg

Akademiestraße 24, A-5020 Salzburg

ulrich.theissen@sgb.ac.at 